\title{
Prototype Design and Application of a Semi-Circular Automatic Parking System
}

\author{
Ismail Atacak*1 and Ertugrul Erdogdu ${ }^{2}$
}

\begin{abstract}
Accepted : 19/12/2017 Published: 28/12/2017
Abstract: Nowadays, with the increasing population in urban settlements, the number of vehicles used in traffic has also increased in these settlements. This has brought with it major problems that are caused by insufficient parking lots, in terms of traffic congestion, drivers and environment. In this study, in order to overcome these problems, a multi-stored automatic parking system that automatically performs vehicle recognition, vehicle parking, vehicle delivery and pricing processes is designed and the practical application of this system is realized on a prototype. The vehicle recognition process in the designed system is fulfilled through a software prepared on the personal computer connected to the webcam. A multi-stored semi-circular structure has been used as parking lots to resolve parking lot deficiencies. Therefore, the carrying system that carries out the parking process in the system is designed as a cylindrical coordinated robot that can move horizontally, vertically and in the forward-back direction. The control of the whole system is realized by the PIC16F877A microcontroller. The results obtained from the prepared prototype have showed that the proposed system can provide significant contributions to the solution of problems resulting from parking lot deficiencies.
\end{abstract}

Keywords: Automatic parking system, cylindrical coordinated robot, position control algorithm, optimal parking lot selection algorithm, plate recognition.

\section{Introduction}

The increase in urbanization in developing countries since the beginning of the 20th century has brought many problems in these settlements. Increased industrialization with the development of technology has led to an increase in population density because of increased migration from rural settlements to urban settlements. As a result, the number of vehicles used in urban settlements has increased and the available parking lots are insufficient to park the vehicles. The inadequacies of the area resulting in the increasing population in these settlements also restrained the establishment of parking lots to resolve the mentioned problem. The addition of the problems caused by distorted urbanization such as business places, inadequate roads and inadequate parking lots in business places has made this problem an indispensable problem, especially in large cities $[1,2]$.

Inadequacies of parking lot cause serious problems on traffic, drivers, environment and national economy [3, 4]. Drivers who can not find a parking lot either increase the traffic density to hit the roads by searching a free parking lot or cause traffic congestion by parking on the roadside [5]. The fact that the drivers can not find a parking lot in the places that they are going to disappoints them and causes them to wear out psychologically. The other effects on the drives can be given as the increase in fuel consumption cost and the waste of time [6-8]. The traffic density or congestion resulting in inadequacies of parking lot increases environmental pollution by raising the fuel consumption [9]. This will also damage the national economy as it will improve health problems based on environmental pollution.

\footnotetext{
${ }^{1}$ Computer Eng., Technology Fac., Gazi Univ. Ankara-06500, TURKEY

${ }^{2}$ Elect. and Comp.Edu.,Inform. Inst., Gazi Univ.Ankara-06500, TURKEY

* Corresponding Author: Ismail AtacakEmail: iatacak@gazi.edu.tr
}

The studies made on both individually and institutionally solving this problem with significant impact on society has made a rising progress in recent years. The solutions proposed as intelligent automatic parking system among them have produced very successful results in reducing the problems mentioned above. Intelligent automatic parking systems represent multi-storey structures consisting of elements equipped with advanced technologies and automatically performing all the processes related to the parking process of vehicles [10]. Because, in these systems, the whole process is performed automatically through the hardware made up the electronic and mechanical structure, some problems mentioned above is completely removed while the rest is minimized. Nowadays, in many cities around the world, such systems have become available to solve the parking problems in crowded urban settlements. There are many studies presented in this sense as prototypes in the literature. Most of these studies correspond to the systems that perform the park process using very few human initiatives. The studies, including the titles of "intelligent parking space detection system based on image processing" [11], "vision based automated parking system" [12], "fuzzy based automatic multi-level vehicle parking using lab view" [13], "efficient automated smart parking system using IOT Technologies" [14] and "PLC based automatic intelligent car parking system" [15], represent some of these studies.

In this study, in parallel with these developments, a semi-circular automatic parking system that automatically performs all operations related to the park process after completing the plate recognition process through a webcam is designed and its application is implemented on a prototype. This paper is organized as follow. In Sec. 2, the block diagram showing the structure of the proposed system is given and its operation is explained through the components making up the system. Sec. 3 presents the design of 
all the components making up the proposed system in detail. Sec. 4 explains the control of the proposed system and the algorithms used in this process. The results obtained from the proposed system are discussed in Sec. 5. In the last section, the performance of the proposed system is evaluated according to these results.

\section{Semi-Circular Automatic Parking System}

The proposed system is an intelligent parking system that automatically carries out the operations including plate recognition, movement from the parking lot to the acceptance/delivery lot or vice versa, and pricing according to the length of stay in the parking lot for the vehicles entering to the acceptance/delivery lot. As understood from the block diagram in Fig. 1, the automatic parking system fulfills the above-described functions using five basic units: a vehicle recognition unit, a serial communication interface, a vehicle carrying unit, a control unit and a two-storied semi-circular building.

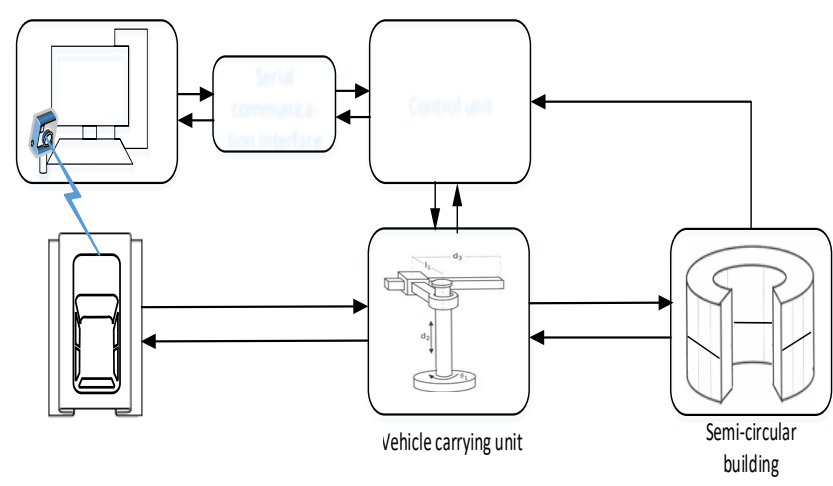

Fig. 1. Block diagram of the semi-circular automatic parking system.

The operation of the system can be explained with two operating mode: vehicle acceptance mode and vehicle delivery mode. The vehicle acceptance mode is put into effect when a vehicle entry takes place to the vehicle acceptance/delivery lot. When this mode is activated, if there is an empty parking lot in the Semi-circular building, firstly, the plate recognition of vehicle is performed through the personal computer connected to the camera in the vehicle recognition unit. The operator then sends a confirmation code from the personal computer to the control unit to start the parking process. After detecting the parking lot closest to the starting position within the empty parking lots as the lot to be parked, the control unit receiving the confirmation code, by executing the position control algorithm, allows that the vehicle carrying unit gets the vehicle from the vehicle acceptance/delivery lot and then parks it in the detected parking lot. After this, while the vehicle carrying unit returns to its starting position, the control unit completes the vehicle acceptance mode process by sending the information about the park operation to the vehicle recognition unit.

The system puts the vehicle delivery mode into effect when the driver comes to the vehicle acceptance/delivery lot to receive his vehicle. After the driver notifies the plate number of his vehicle to the vehicle recognition unit, the parking information registered to the relevant plate, with operator approval, is sent to the control unit through the serial communication interface. Then, the control unit executes the position control algorithm in accordance with the incoming parking information and allows that the vehicle carrying unit moves the vehicle to the vehicle acceptance/delivery lot by getting it from the parked lot. As a final operation, the vehicle recognition unit completes the vehicle delivery mode process by calculating the parking fee for the vehicle.

\section{Design of Proposed System}

The automatic parking system described above its structure and operation is designed as a prototype that is scaled realistically. The simulation of vehicles coming into and leaving the park are made through toy cars. This section explains the designs of four basic components constituting the system, including the parking lots, vehicle carrying unit, electronic equipment and vehicle recognition unit.

\subsection{Parking Lots}

In the design of the parking lots in the prototype to be formed, a multi-storied semi-circular building is preferred due to the fact that it angularly gains from the used area. This building consists of two floors and has 7 parking lots on each floor. 13 vehicles can park to the building with 14 parking lots because the parking lot located in the center of the $1 \mathrm{st}$ floor is used as the vehicle acceptance/delivery lot. The toy cars with dimensions of $14 \mathrm{~cm} \times 10 \mathrm{~cm} \times 10 \mathrm{~cm}$ is used as the vehicle in the prototype. The dimensions of parking lots to be used for parking toy cars are decided by considering the ratio between the volume of the vehicle calculated according to the dimensions of an average vehicle and the volume of the parking lot calculated according to the dimensions of parking lot where this vehicle will park. Table 1 shows the dimensions of an average vehicle and its parking lot in a real parking process.

Table 1. Dimensions of an average vehicle and its parking lot in a real parking process.

\begin{tabular}{lcl}
\hline & Parking lot & Vehicle \\
\hline Length & $1000 \mathrm{~cm}$ & $500 \mathrm{~cm}$ \\
Width & $500 \mathrm{~cm}$ & $280 \mathrm{~cm}$ \\
Height & $500 \mathrm{~cm}$ & $280 \mathrm{~cm}$ \\
\hline
\end{tabular}

According to these data, the ratio between vehicle volume and parking lot volume for an average vehicle $\left(\eta_{\text {Volume }}\right)$ is found as follow:

$\eta_{\text {Volume }}=$ Vehicle volume $/$ Parking lot volume $=0.1568$

It can be concluded from the data in Table 1 that the width and height of parking lot for an average vehicle are equal to each other and its length should be 2 times of the width or height. In this case, the dimensions of the parking lot for an average vehicle can be expressed as 2yxyxy. However, since the parking procedure is carried out by the carrying robot in the designed system, a parking lot length of $1.125 \mathrm{y}$ is sufficient to ensure that the vehicle can be safely parked. Thus, in the designed prototype, the parking lot dimensions for each vehicle are defined to be 1.125 yxyxy. The dimension values for the parking lots in the designed prototype can be obtained as given in Table 2 if the corresponding dimension information and the dimension information of toy car of $14 \mathrm{~cm} \times 10 \mathrm{~cm} \times 10 \mathrm{~cm}$ are written in Equation 1.

Table 2. Dimensions of the toy vehicle used in parking process and its parking lot.

\begin{tabular}{lcc}
\hline & Parking lot & Vehicle \\
\hline Length & $22.5 \mathrm{~cm}$ & $14 \mathrm{~cm}$ \\
Width & $20 \mathrm{~cm}$ & $10 \mathrm{~cm}$ \\
Height & $20 \mathrm{~cm}$ & $10 \mathrm{~cm}$ \\
\hline
\end{tabular}


Because the building with the parking lots is designed as a semicircular form, the total parking lot on each floor corresponds to an angular area of 180 degree. Since there are 7 parking lots on each floor of two-storied building, a parking lot of $180 / 7=25.7$ degrees is allocated for each vehicle. Fig. 2 shows the preview image of the first floor of the prototype designed according to these data.

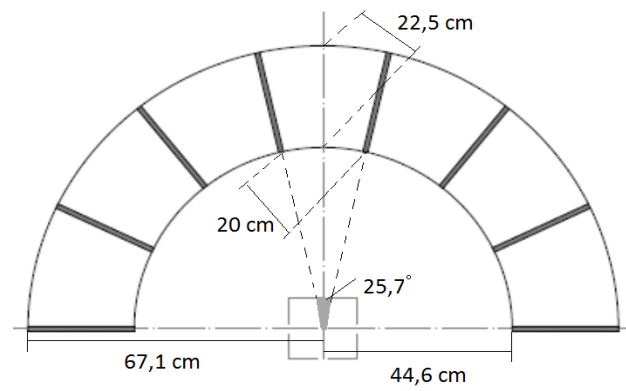

Fig 2. Preview image of the first floor of the prototype designed according to the calculated data.

In the installation of the designed building, the PVC foam plates are utilized since it is easy to handle, light and durable. The installation is started from the base and an aluminum composite plate with thickness of $5 \mathrm{~mm}$ is used for the bottom layer which will carry the parking compartments and the Vehicle carrying unit. Fig. 3 shows the picture of two-stored semi-circular building completed the installation in accordance with the preview image given in Fig. 2.

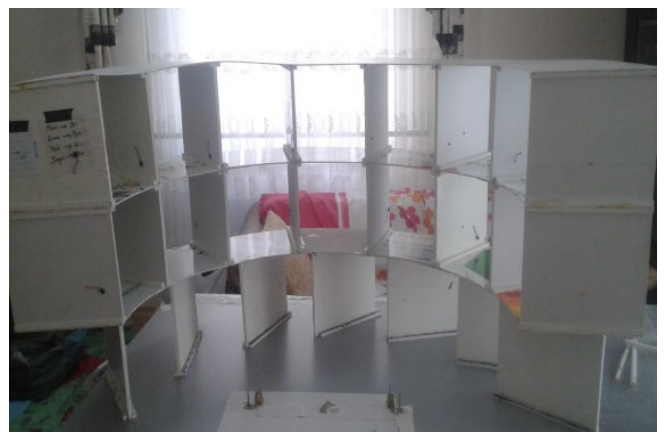

Fig. 3. Semi-circular building with completed installation

\subsection{Vehicle Carrying Unit}

A cylindrical coordinated robot that can move in horizontal, vertical and forward-backward directions is preferred as the vehicle carrying unit because the parking process is carried out on a semi-circular building. The cylindrical coordinated robot system is made of a sheet metal with thickness of $1.5 \mathrm{~mm}$ and includes a mechanical structure comprising stepper motors, gears, a trigger belt, rails, an axial deep groove ball bearing, and stationary and movable sheet metals. The installation of the robot system is started from the base bearing all the load and the sheet metal base with the dimension of $30 \mathrm{~cm} \times 30 \mathrm{~cm}$ and the high of $3 \mathrm{~cm}$ from the ground by bending from the edges is obtained. A tower with the dimension of $75 \mathrm{~cm} \times 10 \mathrm{~cm} \times 11 \mathrm{~cm}$ is built on the base.

As shown in Fig. 4, the mechanism which provides vertical movement consists of drawer rails, metal components moving in the rail, a stepper motor and a belt gear system. To create vertical movement path, the drawer rails are mounted on the left and right side of the inner part of the tower. Two vertical holes of $48 \mathrm{~cm}$, parallel to each other, are drilled on the front face of the tower in order to link the palette system carrying the vehicle with the carrying arm that combines the parts used in the horizontal and back-and-forth movement. In order to ensure vertical movement, two metal parts, named as A and B, are placed in the drawer rails. A gear belt system which is connected to a stepper motor placed at the bottom left of the tower is used as the source of vertical movement. The trigger belt is fixed to A and B metals so that the vertical movement can be provided by the movement of the gear belt system.
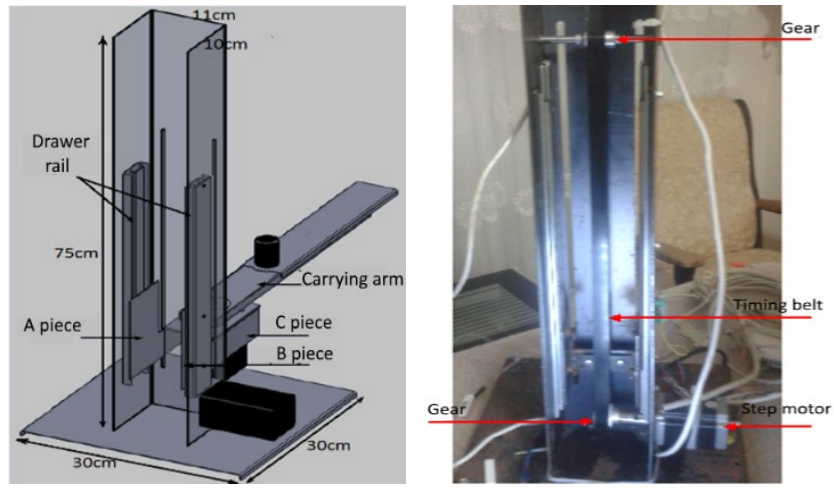

Fig. 4. Vertical movement elements for cylindrical coordinated robot system: a) fixed and movable metal elements b) gear belt system

The mechanism providing horizontal movement is placed in the front of the tower. As shown in Fig. 5, this mechanism consists of a metal piece called $\mathrm{C}$, an aluminum piece called $\mathrm{D}$, an axial deep groove ball bearing and a step motor. In this structure, $\mathrm{C}$ metal is used both to link the metals A and B in the vertical movement mechanism and to carry the vehicle carrying arm, while the aluminum $\mathrm{D}$ is used to extend the surface of the metal $\mathrm{C}$. The rotational movement of $0-180$ degrees in horizontal motion is achieved by the stepper motor fixed on the parts $\mathrm{C}$ and $\mathrm{D}$ which are interlocked each other. In the horizontal cylindrical movement, the axial deep groove ball bearing is placed between the motor shaft and the vehicle carrying arm to reduce friction and prevent fully riding of the load on the motor shaft. The movement of the vehicle carrying arm is provided by connecting this arm to the motor shaft.

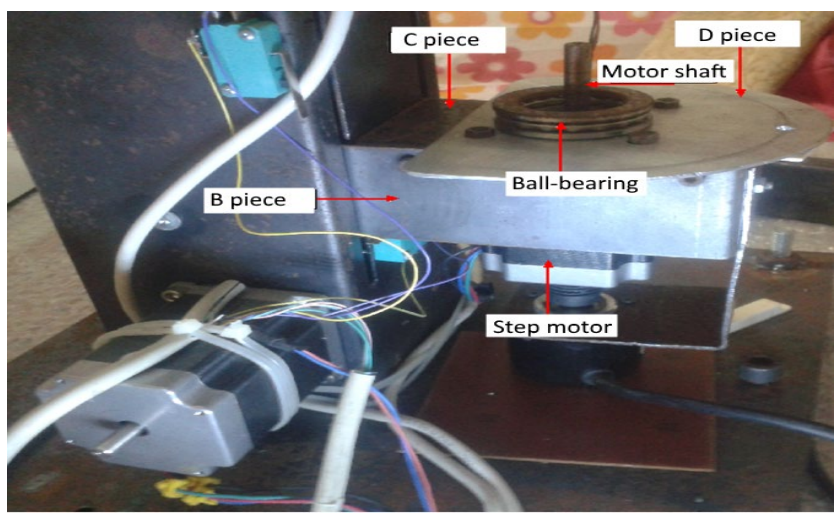

Fig. 5. Horizontal movement elements

The mechanism providing the back-and-forth movement includes a palette and a low-torque stepper motor placed on the vehicle carrying arm. As shown in Fig. 6, the palette consists of the combination of a U-shaped bottom groove and a reversed Ushaped top groove. Where while the bottom groove is formed by bending $1.5 \mathrm{~cm}$ from the edges of a metal with the dimension of $40 \mathrm{~cm} \times 8 \mathrm{~cm}$, the top groove is composed by bending $1 \mathrm{~cm}$ from the 
edges of another metal with the dimension of $30 \mathrm{~cm} x 8 \mathrm{~cm}$. To create the path of back-and-forth movement, a rack-bar is placed in the bottom groove. In order to enable the back-and-forth movement, the stepper motor is engaged on the top groove and a pinion gear moving on the rack-bar is fixed to the motor shaft.

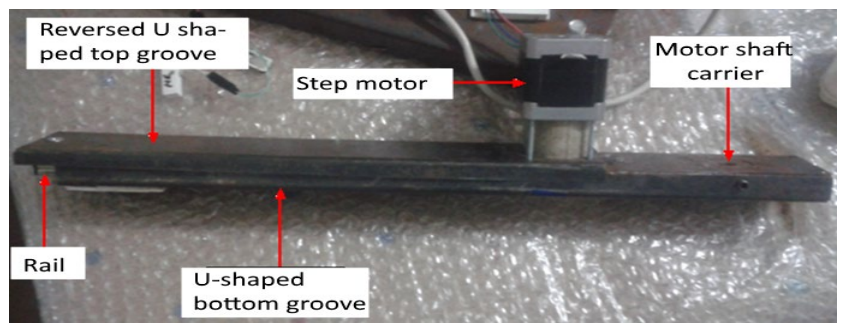

Fig. 6. Palette elements.

\subsection{Electronic Circuit}

As shown in Fig. 7, the electronic circuit of the automatic parking system consists of 5 basic components including a microprocessor, sensors, step motors, step motor drivers, and a serial communication interface.

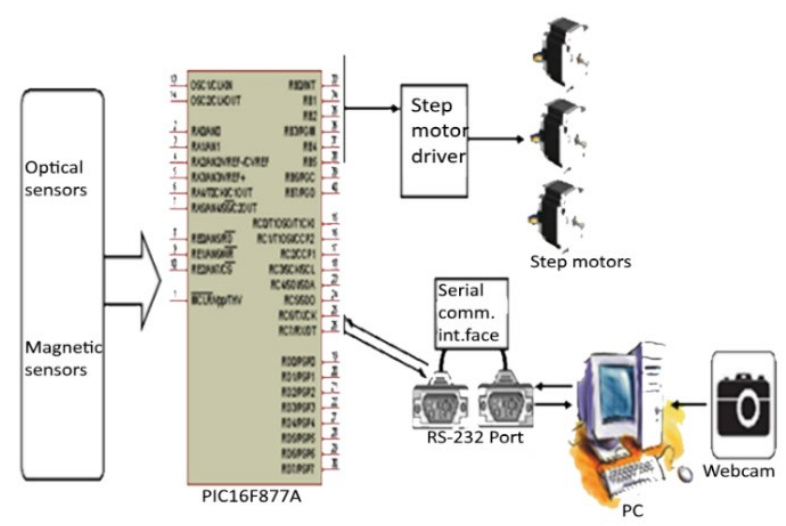

Fig. 7. Components making up the electronic circuit of the automatic parking system.

The sensors, which are used to measure the state information about the parking lots and the vehicle carrying arm, are decided in the first step of electronic circuit design. Two type sensors are used to fulfill the mentioned functions in the system: magnetic sensor and optical sensor. The state information related to whether parking lots are empty or not is detected by optical sensors, and the Sharp GP2Y0D810Z0F digital infrared proximity sensors are preferred as optical sensors due to the fact that they have short sensing range and fast response time. The magnetic sensors, known as reed relays, are used in detecting the state information about the backand-forth movement limits of the vehicle carrying arm. The HE3300 series miniature single in line reed relays are preferred as magnetic sensors in the system since it minimizes space and cost.

In the second step of electronic circuit design, the selection of the step motors mounted on the vehicle carrying unit and the drivers used to drive these motors are decided. The selection of step motors operating as the component that moves the arm in the vehicle carrying unit is made according to the torque needed for the movement in horizontal, vertical and forward-backward directions. In order to obtain the torque required in the all directions, the force of the weight on the motor shaft and its distance to the motor shaft (force arm length) are needed to be known. Table 3 shows the weights and force arm lengths measured on the shafts of the step motors used in vertical, horizontal and back-and-forth movements, and the torques calculated according to these values.

Table 3. The torques calculated for vertical, horizontal and back-and-forth movements.

\begin{tabular}{lccc}
\hline Movement types & $\begin{array}{c}\text { Weights } \\
\text { on the motor } \\
\text { shaft (gr) }\end{array}$ & $\begin{array}{c}\text { Force } \\
\text { arm } \\
\text { lengths (m) }\end{array}$ & $\begin{array}{c}\text { Calculated } \\
\text { torques (gr.m } \\
\left.\text { or gr.m }^{2}(*)\right)\end{array}$ \\
\hline Vertical, movement & 3600 & 0.02865 & 128.928 \\
Horizontalmovement $\left(^{*}\right)$ & 2200 & 0.4 & 146.625 \\
$\begin{array}{l}\text { Back-and-forth } \\
\text { movement }\end{array}$ & 200 & 0.03 & 7,5 \\
\hline
\end{tabular}

The market surveys on step motors shows that the SY57STH762804A (NEMA23) model step motors can meet the calculated torque and inertia force in the vertical and horizontal movement while the SY42STH47-1684A (NEMA17) model step motors can meet the calculated torque and inertia force in the back-and-forth movement. The ZM-2H504 step motor driver, which is designed to operate with 2, 4 or 8-phase step motor and with maximum current per phase up to $4.2 \mathrm{~A}$, is used to drive the NEMA model step motors.

The communication protocol and interface circuit that communicates between the microcontroller and the vehicle recognition unit are determined in the third step of the electronic circuit design. Personal computers use three type ports for communication with peripherals: a parallel (LPT) port, a serial (COM) port and a universal serial bus (USB) port. The communication between the microcontroller and the personal computer in the system is made on the COM port because it has a less data communication line than the other ports. The distance between the personal computer and the microcontroller in the system is $70 \mathrm{~cm}$. RS-232 protocol is used as serial communication protocol since it can provide lossless data communication at less than $20 \mathrm{~m}$. Due to the fact that the microcontroller is an integrated TTL circuit, the voltage levels at its receiver and transmitter pins are different from those of the personal computer Therefore, it is not possible to communicate directly between the personal computer and the microcontroller by using this protocol. A voltage-matching circuit between both units is required to communicate. The MAX-232 integrated circuit is used to achieve this purpose in this system.

The final step of electronic circuit design is the choice of microcontroller that performs control of the whole system. This process is conducted considering the system requirements and cost criteria. In order to fulfill the control function in the designed system, we need 18-programmable digital port inputs to receive the information from sensors, 6-programmable digital port outputs to drive the step motors and 2-serial communication input/output ports to communicate with the personal computer. The market surveys on microcontrollers show that the PIC16F877A microcontroller is the most suitable controller to be used in the control of the proposed system in terms of both system requirements and cost.

\subsection{Vehicle Recognition Unit}

The structure of this system, which communicates with the PIC16F877A microcontroller in the control process of automatic parking system, constitutes a personal computer, a simple WEBCAM and a computer software. In this structure, the computer software is the component that performs the basic function of the vehicle recognition unit. This software is a singleuser interface program that allows the automatic parking system 
automatically to take a photo of the vehicles entering to the acceptance/delivery lot, to perform the plate recognition process of the vehicle through this photo, to save information about the vehicles and the entrance and exit to the parking lot into the database, to calculate the parking charge according to the time when vehicles are situated in the parking lot and to make data exchange with the microcontroller during the control process. The interface program is written in the $\mathrm{C} \#$ programming language and made functional all computers, on which the Microsoft Windows operating system is installed, after a setup file is created. The Microsoft Access, developed by Microsoft Corporation, is used as a database management program because it is reliably being run on all computers on which the Microsoft Office programs are installed and the saving process does not needed a very large data stack area. Optical character recognition (OCR) technology is used for the plate recognition process in the interface program. In practice, there are several OCR programs, such as ABBYY FineReader, Tesseract, and Microsoft Office Document Imaging (MODI), which convert to text form by selecting letters from the image files taken from scanner or camera. In this study, the MODI, which is a free program in Microsoft Office program, is utilized as a OCR technology program in the plate recognition process. Preview image of the interface program developed for the vehicle recognition unit when running the software is shown in Fig. 8.

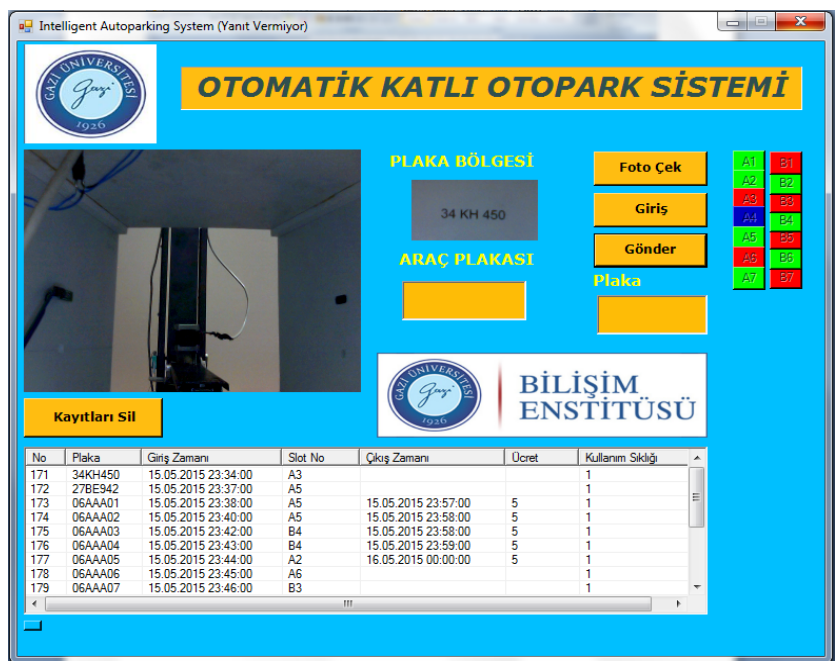

Fig. 8. Preview image of the interface program developed for the vehicle recognition unit.

The operation of the interface program can be explained as follows: when the program is first run, it queries the information about whether the vehicle carrying unit is ready to operate or not through the serial communication interface from the microcontroller. If the carrying system is ready to operate, the microcontroller sends the ready information $(\mathrm{X})$ to the vehicle recognition unit. The vehicle recognition unit that receives the information displays the database records on the screen and makes the camera ready for receiving an image. When a vehicle entry takes place to the vehicle acceptance/delivery lot the microcontroller sends the photo check command (Q) to the vehicle recognition unit depending on the state information received from the infrared proximity sensors. The vehicle recognition unit that receives this command sends the command that initiates the parking process $(\mathrm{W})$ with operator approval to the microcontroller after performing respectively the photo shoot and the plate recognition processes of vehicle. The microcontroller sends parking lot information to the vehicle recognition unit after completing the parking process of the vehicle according to the incoming command. This system completes the process by saving the information on the plate number, parking time and parking lot into the database.

When a driver comes to receive his/her vehicle to the vehicle acceptance/delivery lot, the operator enters the plate number from him into the interface program and sends it to the microcontroller through the serial interface by pressing the "Send" button. The microcontroller initiates the exit process from the parking lot of vehicle with this information and sends the process completed information $(\mathrm{W})$ to the vehicle recognition unit when the process completes. The vehicle recognition unit calculates the parking charges for the vehicle leaving the park as soon as it receives this information and then completes the process by updating the information about the parking operations of vehicle.

In calculating the parking charges for a parked vehicle in the automatic parking system, three parameters are considered, namely the base charges $\left(C_{\text {base }}\right)$, the duration of time at the park $\left(t_{d u r}\right)$, the hourly parking charges $\left(C_{h}\right)$ and the discount rate $\left(\% \eta_{\text {disc }}\right)$. The equation used in the calculation of the parking charges $\left(C_{\text {parking }}\right)$ according to these parameters is given below.

$$
C_{\text {parking }}=\left[C_{\text {base }}+\left(t_{\text {dur }}-1\right) \cdot C_{h}\right] \cdot \frac{\left(100-\% \eta_{\text {disc }}\right)}{100}
$$

Discount rate $\% \eta_{\text {disc }}$ is a parameter whose value ranges from 0 to 40. In this study, fuzzy logic (FL) algorithm is used to determine the value of the relevant parameter. When determining the discount rate for a vehicle by the FL algorithm, two basic criteria is taken into consideration: one is the usage frequency of parking lots by vehicle and the other is the total usage time of parking lots by vehicle. Therefore, the usage frequency and total usage time of parking lots are selected as the input variables. Each input variable is represented by three different fuzzy sets, whose name are "LITTLE" (LI), "MEDIUM" (ME), and "MUCH" (MU). The triangular type membership functions are used to define these fuzzy sets. Fig. 9 show the triangular type membership functions defined for the usage frequency and total usage time input variables, respectively.

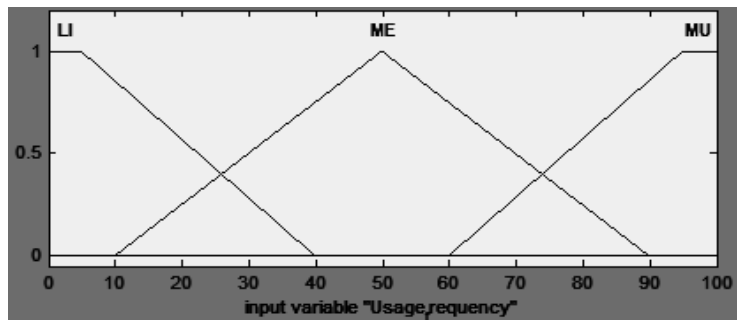

(a)

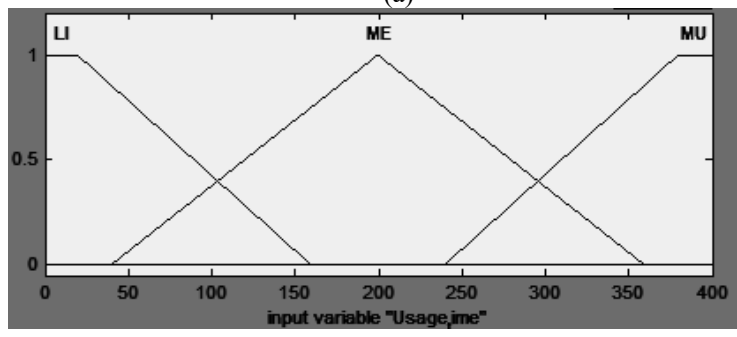

(b)

Fig. 9. Triangular type membership functions defined for the input variables: a) for the usage frequency of parking lots, b) for the total usage time of parking lots.

The discount rate used as the output variable is represented by five fuzzy sets: "VERY LITTLE" (VLI), "LITTLE" (LI), "MEDIUM" 
(ME), "MUCH" (MU), and "VERY MUCH" (VMU). These fuzzy sets are defined by the triangular type membership functions, as being in the input fuzzy sets. The triangular membership functions defined for the discount rate output are shown in Fig.10.

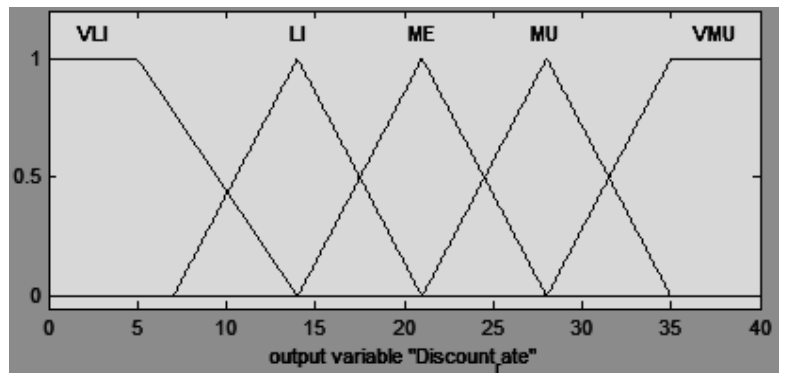

Fig. 10. Triangular type membership functions defined for the discount rate output

Since each input of the FL algorithm with two inputs has 3 fuzzy sets, the rule base consists of 9 rules. Table 4 shows these rules formed for the FL algorithm.

Table 4. Rule base for the discount rate output.

\begin{tabular}{cllll} 
& \multicolumn{4}{c}{ Usage frequency input } \\
\cline { 2 - 5 } Usage & LI & LI & ME & MU \\
time & ME & VLI & LI & ME \\
input & MU & ME & ME & MU \\
& & & MU & VMU
\end{tabular}

The inference process of FL algorithm are conducted through the max-min inference strategy which is known to produce a more efficient inference result than other inference strategies. The weighted average method is used in the defuzzification process because it both has less computational intensity and produces a results close to the centroid method which gives the most accurate result among the available methods.

\section{Control of Proposed System}

The control of the automatic parking system is carried out through the program written in the PIC16F877A microcontroller. The flowchart of this program is given in Fig. 9. This program implements two procedures according to the information coming from the vehicle recognition unit. When entering a vehicle to the acceptance/delivery lot, the parking process is carried out through the first procedure. The relevant procedure completes the parking process by executing the subroutines including the "Optimal Parking Lot Selection" (OPLS) and "Position Control" (POC) algorithms, respectively, after detecting the free parking lots according to the information obtained from the infrared proximity sensors. In the case that the information from the vehicle recognition unit is plate information, the second procedure is implemented to fulfill the exit process of a vehicle located in parking lot. This procedure carries out the exit process by executing the subroutine of the POC algorithm after detecting the recorded parking lot according to the incoming plate information.

As understood from the flowchart of the program, two different algorithms are used in order to fulfill the process related to the control of the automatic parking system. The OPLS algorithm, the first one of these algorithms, selects the most suitable parking lot for a vehicle entering to the acceptance/delivery lot. In the proposed system, the transporting velocity is fixed and it is aimed that the parking process to be done quickly with minimum energy consumption. Thus, in the algorithm, the parking lot which has the shortest parking time with respect to the vehicle acceptance/delivery lot (parking lot "14") is considered as the optimal parking lot. We here uses the time elapsed to move the vehicle from the parking lot " 14 " to the selected parking lot in order to get a simpler equation to be used in microcontroller instead of parking time. The Vehicle carrying unit covers the distance between two floors in vertical movement in $5.2 \mathrm{sec}\left(t_{v}\right)$ and the distance between two parking lot in horizontal motion in $2.75 \mathrm{sec}$ $(t h)$.

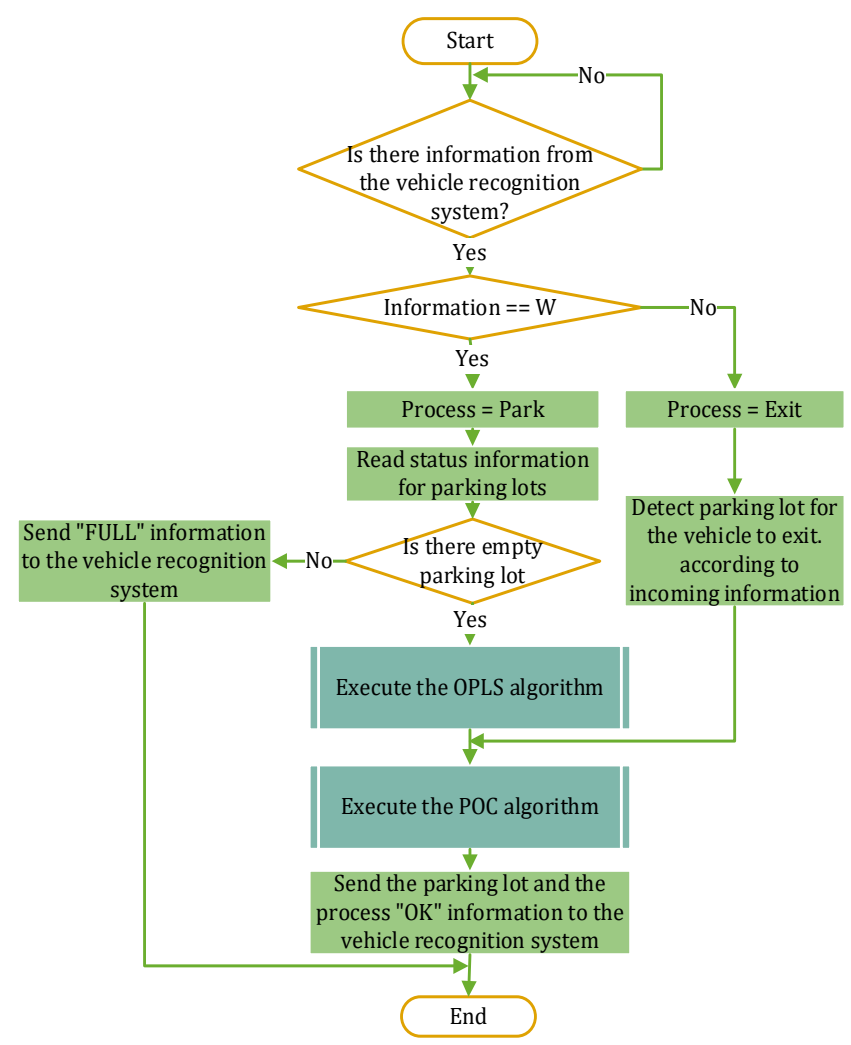

Fig. 9. Flowchart of the program used in the control of the proposed system.

Depending on these data, the time $\left(t_{t p}\right)$ when is needed to move a vehicle from parking lot " 14 " to a free parking lot can be obtained as follow.

$t_{t p}=\left(N_{f n}-1\right) \cdot t_{v}+\left|4-N_{p l n}\right| \cdot t_{h}$

Table 5. Naming the parking lots by floors.

\begin{tabular}{cccccccc}
\hline Floor numbers & \multicolumn{7}{c}{ Parking lot numbers } \\
\hline $2^{\text {nd }}$ floor & 21 & 22 & 23 & 24 & 25 & 26 & 27 \\
$1^{\text {st }}$ floor & 11 & 12 & 13 & 14 & 15 & 16 & 17 \\
\hline
\end{tabular}

Where $N_{f n}$ is the floor number of the parking lot where the vehicle will be parked, and $N_{p l n}$ is the field number of parking lot where the vehicle will be parked. The OPLS algorithm calculates the elapsed times by applying this equation to each of the free parking lots detected by the infrared proximity sensors in the main program and then it selects the parking lot with the lowest elapsed time among the calculated times as an optimal parking lot. If there are more than one parking lots with the lowest elapsed time, then, the algorithm selects the one which has the lowest floor and parking lot numbers as the optimal parking lot. The flowchart of the OPLS algorithm is shown in Fig. 10. 


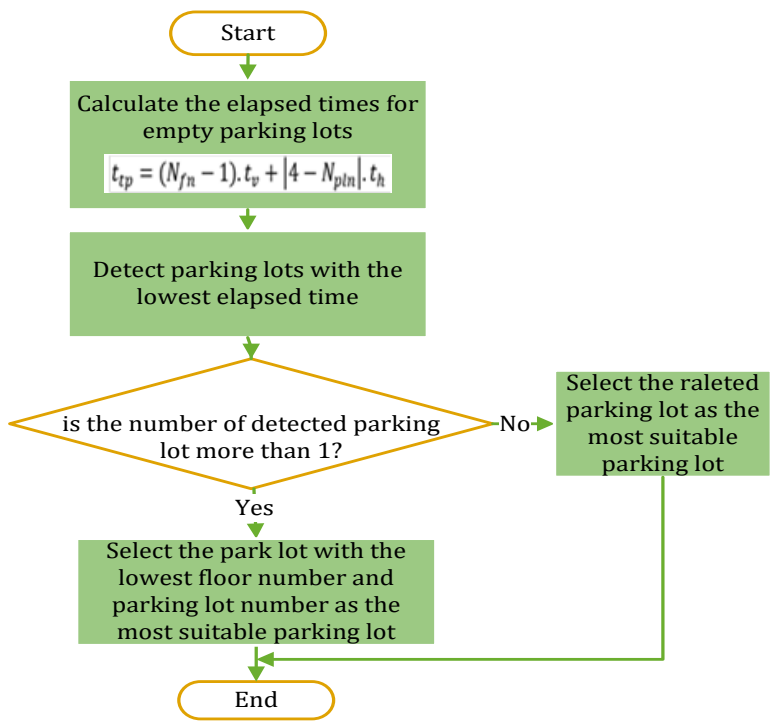

Fig. 10. Flowchart of the OPLS algorithm

The POC algorithm is the second algorithm related to the control of the proposed system which is used to implement the parking entrance and exit processes. This algorithm generates the control signals needed by the stepper motors in the vehicle carrying unit to park a vehicle to the selected parking lot or to move a vehicle from the detected parking lot to the vehicle acceptance/delivery lot. The algorithm performs this function by executing 3 subroutines: backand-forth movement subroutine, vertical movement subroutine and horizontal movement subroutine. Fig. 11 depicts the flowchart of the POC algorithm that shows the order in which the subroutines are executed, depending on the information assigned to the process variable.

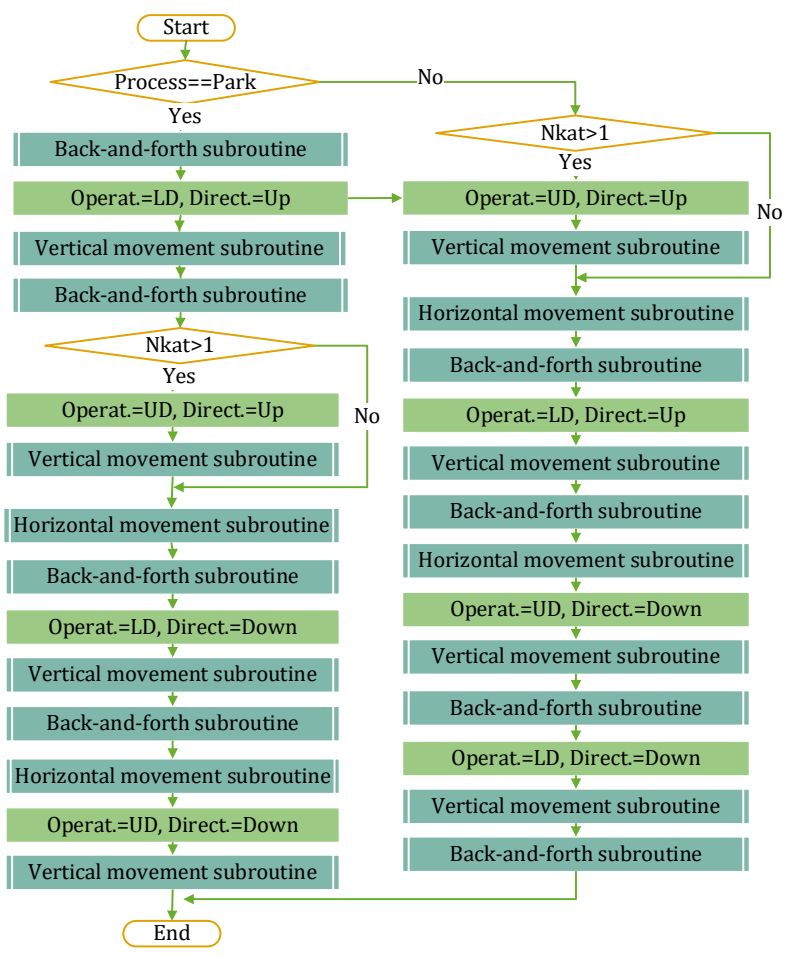

Fig. 11. Flowchart of the $P O C$ algorithm that shows the order in which the subroutines are executed.

The back-and-forth movement subroutine generates the control signals for the step motor used in back-and-forth movement according to the switch position information of the reed relays mounted on the right and left of the pallet on the carrying arm of the vehicle carrying unit. When the switch on the right of the palette (SW1) is on and the switch (SW2) on the left is off, the microcontroller pulls the voltage of RB4 port to the high level so that the pallet can move forward direction and sends the generated clock pulse to the RB5 port to run the step motor until the SW2 switch is on. In the case that the state information of switches is opposite to the previous ones, the microcontroller pulls the voltage of RB4 port to the low level so that the pallet can move backward direction and sends the generated clock pulse to the RB5 port to run the step motor until the SW1 switch is on. The flowchart of the back-and-forth movement subroutine is shown in Fig 12 .

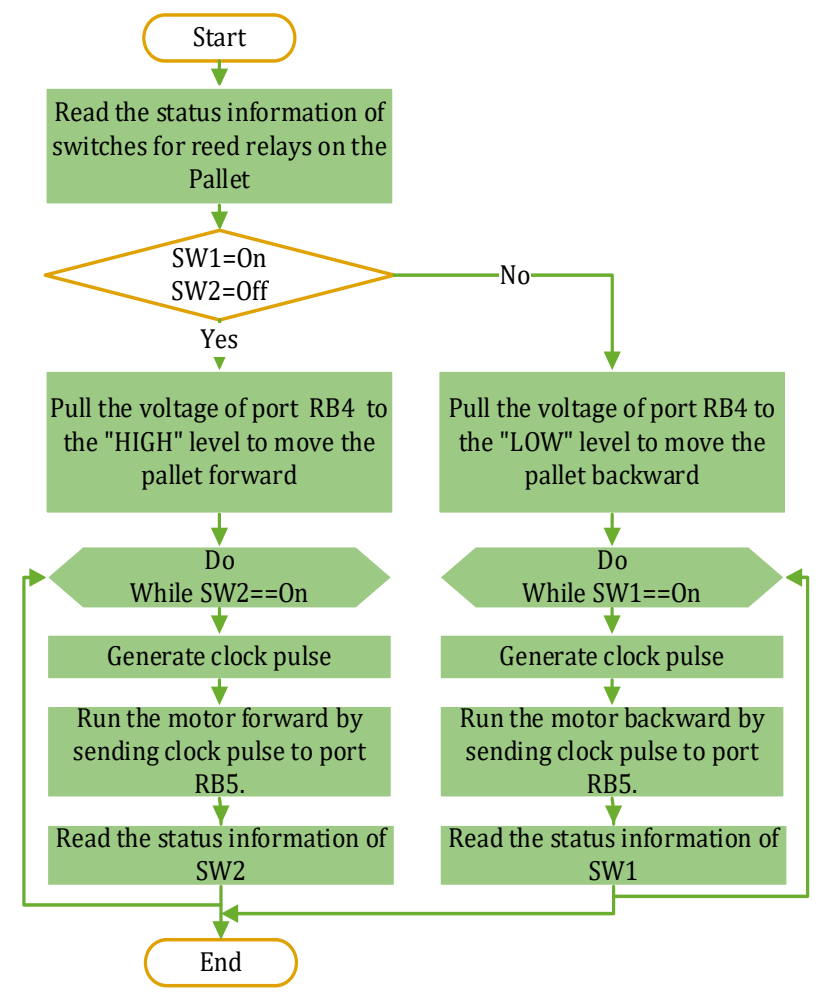

Fig. 12. Flowchart of the back-and-forth movement subroutine

The vertical movement subroutine, with respect to the operation information and direction information determined by the POC algorithm, generates the required control signals for the step motor which is used in vertical movement to perform the processes related to the 1-folding up/1-folding down and setting down/lifting of a vehicle located in the parking lot. As understood from the flowchart in Fig. 13, if "LD" is assigned as the operation type by the POC algorithm the setting down/lifting process of the vehicle in parking lot is put into effect while the 1 -folding up/1-folding down process of the vehicle is implemented in the case of that "UD" is assigned as the operation type. Direction information decides in which direction the movement will be. If this information is assigned as "Up", the movement will be in the upward direction, while if not so it will be in downwards direction. Where the $N_{v s t e p}$ variable decides how many steps the step motor will take depending on the type of assigned operation. The distance to be covered by the motor in the process of setting down/lifting a vehicle is $20 \mathrm{~mm}$ whereas the distance in the one of the1-folding up/1-folding down is $200 \mathrm{~mm}$. The calculations made by taking into consideration the circumference of the gear connected to the motor shaft in vertical movement shows that the motor completes the distance of $1 \mathrm{~mm}$ in 17.77 steps. According to these data, $N_{v s t e p}$ 
is calculated to be 355 steps when "LD" is selected as the process type and it is obtained to be 3555 steps when "UD" is selected as process type.

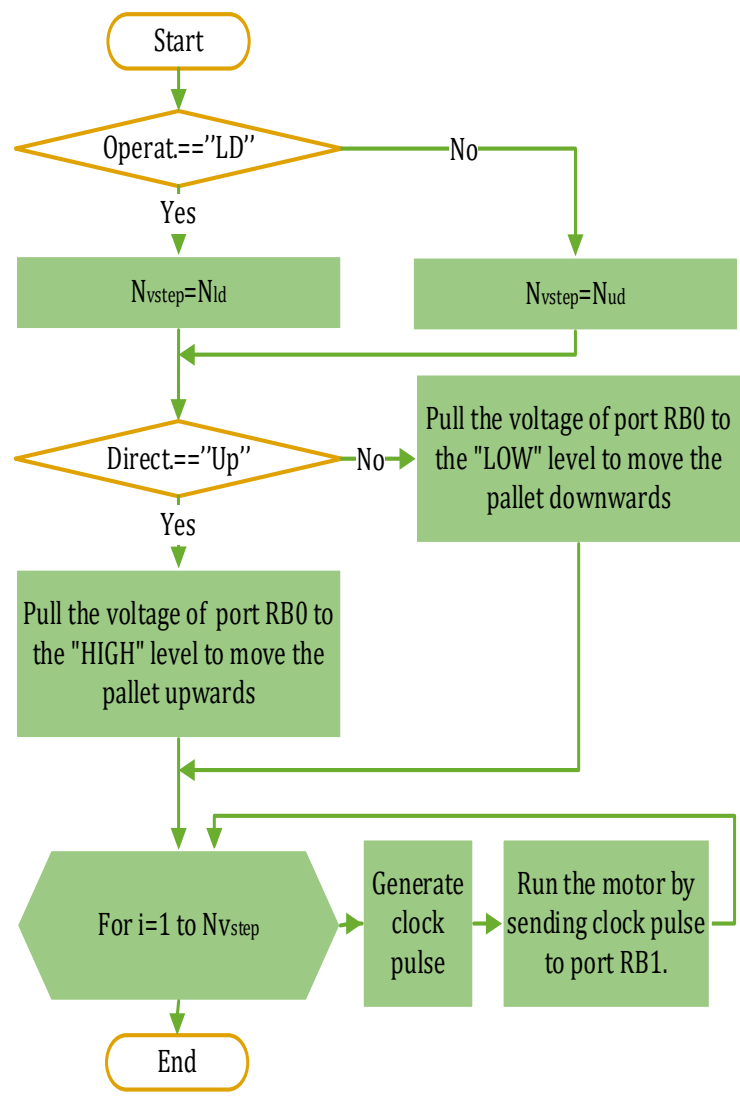

Fig. 13. Flowchart of the vertical movement subroutine

The horizontal movement subroutine calculates the number of steps needed for horizontal movement according to the selected parking lot, and generates the required control signals for the step motor used in this movement. The number of steps needed to reach a selected parking lot in the horizontal movement $\left(N_{h s t e p}\right)$ can be calculated by considering the number of steps corresponding to one degree rotation of the carrying arm $\left(N_{I_{-} d e c}\right)$ and the angle value between two parking lots $\left(\alpha_{p l}\right)$.

$N_{\text {hstep }}=\left|N_{p l}-4\right| . N_{1 \_d e c} . \alpha_{p l}$

Where the $N_{1 \_d e c}$ is determined according to the circumference of the half circle drawing the carrying arm and the catalogue values of the step motor used in the horizontal movement, and this value is calculated as 77.11. The transition angle $\alpha_{p l}$ between parking lots is calculated as $180^{\circ} / 7=25.7^{\circ}$ since the building used as parking lots is designed as half circle and each floor has 7 parking lots in automatic parking system. The direction in the horizontal movement is decided according to the difference between the acceptance/delivery lot number and the selected parking lot number. If this difference is positive, the direction of movement will be towards the left side, otherwise it will be towards the right side. The flowchart of the horizontal movement subroutine is shown in Fig. 14.

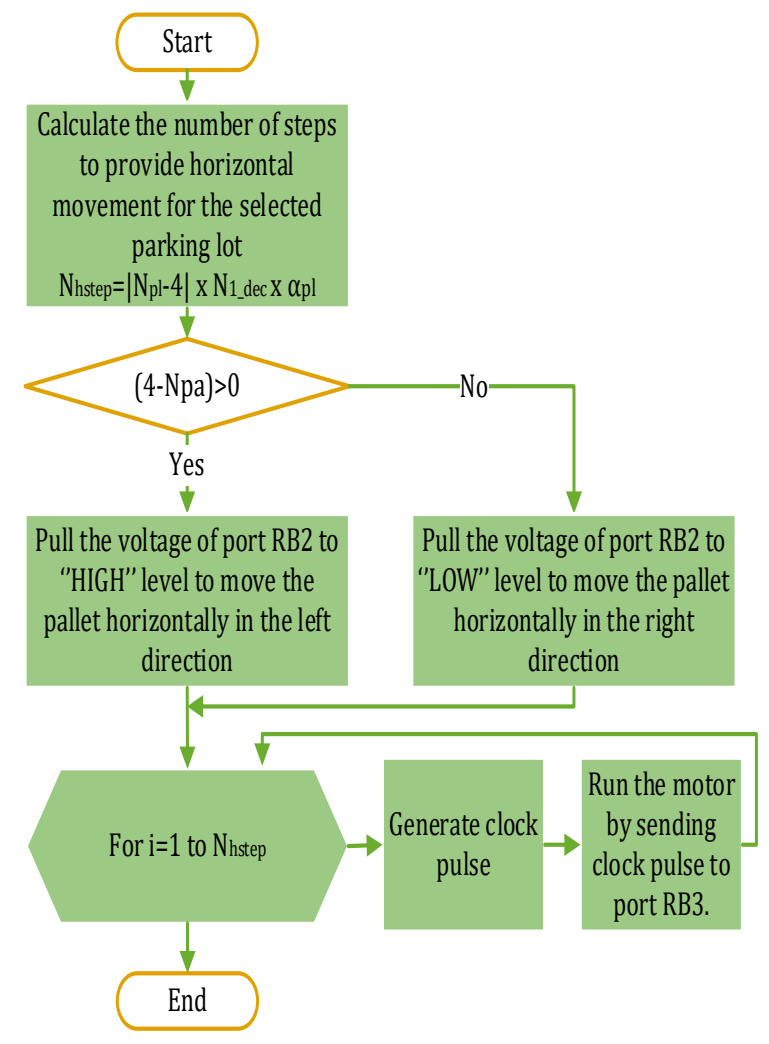

Fig. 14. Flowchart of the horizontal movement subroutine.

\section{Results and Discussions}

The picture of the semi-circular automatic parking system, which is designed by using the components given in Sec. 3 and whose control is realized by using the control algorithms given in Sec. 4, is shown in Fig. 15. The contribution of the proposed system to the solution of parking lot problems is confirmed by considering 4 performance criteria. These are the parking time periods of vehicles, the energy consumption of the proposed system in the parking process, the discount rate in the parking charges for drivers and the area and volume ratios of the automatic parking buildings.

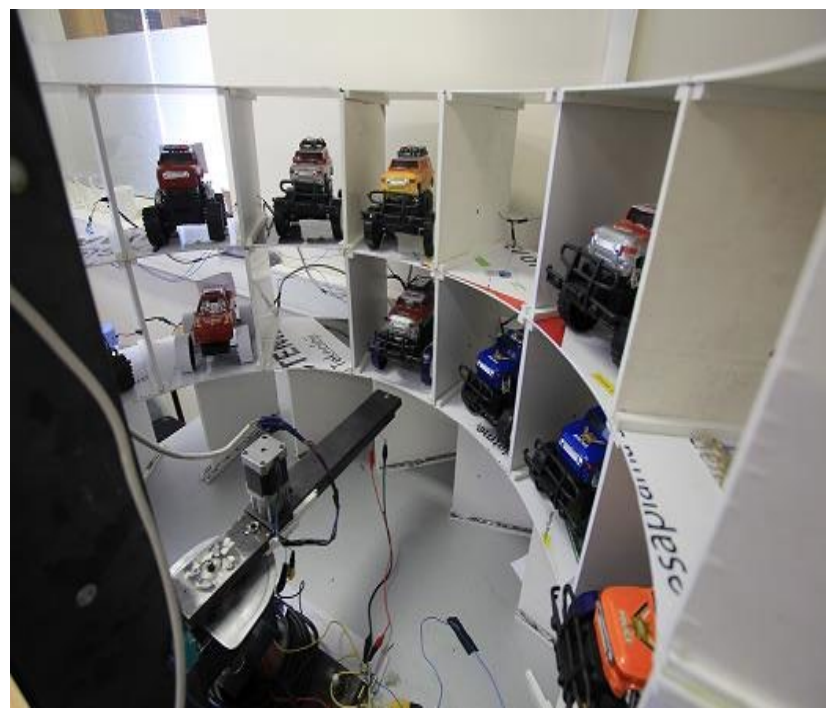

Fig. 15. Picture of the designed semi-circular automatic parking system. 
The parking time period $\left(t_{p p}\right)$ of a vehicle consists of the sum of the elapsed time $\left(t_{p r k}\right)$ until the vehicle carrying unit take the vehicle from the vehicle acceptance/delivery lot and parking it into the selected parking lot, and the elapsed time $\left(t_{r s p}\right)$ to return to its starting position. In order to obtain this time period, we first measure the elapsed times for the completion of back-and-forth $\left(t_{f b}\right)$, lift-and-down $\left(t_{l d}\right)$, horizontal $\left(t_{h}\right)$ and vertical $\left(t_{v}\right)$ movements used in the parking procedure with a timekeeper. Then, the time period for the parking process of a vehicle is obtained by writing the measured values in the equations we obtained from the POC algorithm given in Sec. 4 . The $t_{p r k}$ and $t_{r s p}$ equations obtained from the parking process sequence in the POC algorithm are respectively given by,

$t_{p r k}=\left(N_{f n}-1\right) \cdot t_{v}+\left|4-N_{p l n}\right| \cdot t_{h}+3 t_{f b}+2 t_{l d}$

$t_{r s p}=\left(N_{f n}-1\right) \cdot t_{v}+\left|4-N_{p l n}\right| \cdot t_{h}+t_{f b}$

Where $t_{l d}$ shows the elapsed time for the vehicle carrying unit to lift or down a vehicle. By taking these equations into account, the parking time period can be obtained as $t_{p p}=t_{p r k}+t_{r s p}$. The $t_{p r k}, t_{r s p}$ and $t_{p p}$ time periods, which are calculated according to the $t_{f b}, t_{l d}, t_{h}$ and $t_{v}$ times measured for back-and-forth, lift-and-down, horizontal and vertical movements, is shown in Table 6. It is understood from the data in the table that the parking time period of the parking lots dually numbered "13-15", "12-16", "11-17", "23-25”, "22-26" and "21-27" are equal to each other because their distances from the vehicle acceptance/delivery lot are identical. From these data, it can also be understood that the parking lots with the shortest time period are the "13" and "15" numbered parking lots while the ones with the longest time period are the "21" and "27" numbered parking lots.

Table 6. Parking time periods related to the parking process of a vehicle according to parking lot numbers.

\begin{tabular}{cccccccc}
\hline $\begin{array}{c}\text { Parking } \\
\text { lots }\end{array}$ & $\begin{array}{c}\mathbf{t}_{\mathbf{v}} \\
(\mathbf{s e c})\end{array}$ & $\begin{array}{c}\mathbf{t}_{\mathbf{h}} \\
(\mathbf{s e c})\end{array}$ & $\begin{array}{c}\mathbf{t}_{\mathbf{f b}} \\
(\mathbf{s e c})\end{array}$ & $\begin{array}{c}\mathbf{t}_{\mathbf{l d}} \\
(\mathbf{s e c})\end{array}$ & $\begin{array}{c}\mathbf{t}_{\text {apl }} \\
(\mathbf{s e c})\end{array}$ & $\begin{array}{c}\mathbf{t}_{\mathbf{r s p}} \\
(\mathbf{s e c})\end{array}$ & $\begin{array}{c}\mathbf{t}_{\text {prk }} \\
(\mathbf{s e c})\end{array}$ \\
\hline 11 & 0.00 & 2.75 & 2.50 & 0.54 & 15.75 & 10.75 & 26.50 \\
12 & 0.00 & 2.75 & 2.50 & 0.54 & 13.00 & 8.00 & 21.00 \\
13 & 0.00 & 2.75 & 2.50 & 0.54 & 10.25 & 5.25 & 15.50 \\
14 & 0.00 & 0.00 & 0.00 & 0.00 & 7.50 & 0.00 & 0.00 \\
15 & 0.00 & 2.75 & 2.50 & 0.54 & 10.25 & 5.25 & 15.50 \\
16 & 0.00 & 2.75 & 2.50 & 0.54 & 13.00 & 8.00 & 21.00 \\
17 & 0.00 & 2.75 & 2.50 & 0.54 & 15.75 & 10.75 & 26.50 \\
21 & 5.20 & 2.75 & 2.50 & 0.54 & 20.95 & 15.95 & 36.90 \\
22 & 5.20 & 2.75 & 2.50 & 0.54 & 18.20 & 13.20 & 31.40 \\
23 & 5.20 & 2.75 & 2.50 & 0.54 & 15.45 & 10.45 & 25.90 \\
24 & 5.20 & 2.75 & 2.50 & 0.54 & 12.70 & 7.70 & 20.40 \\
25 & 5.20 & 2.75 & 2.50 & 0.54 & 15.45 & 10.45 & 25.90 \\
26 & 5.20 & 2.75 & 2.50 & 0.54 & 18.20 & 13.20 & 31.40 \\
27 & 5.20 & 2.75 & 2.50 & 0.54 & 20.95 & 15.95 & 36.90 \\
\hline
\end{tabular}

Fig. 16 shows the changes in the parking time period according to the occupancy rate of parking lots. It can be clearly seen from the graphic that the parking time period of a vehicle also increases as the occupancy rate of parking lots increases. The parking time period is $15.5 \mathrm{sec}$ when the occupancy rate is $0 \%$ whereas it increases to $36.9 \mathrm{sec}$ when the rate reaches $85 \%$.

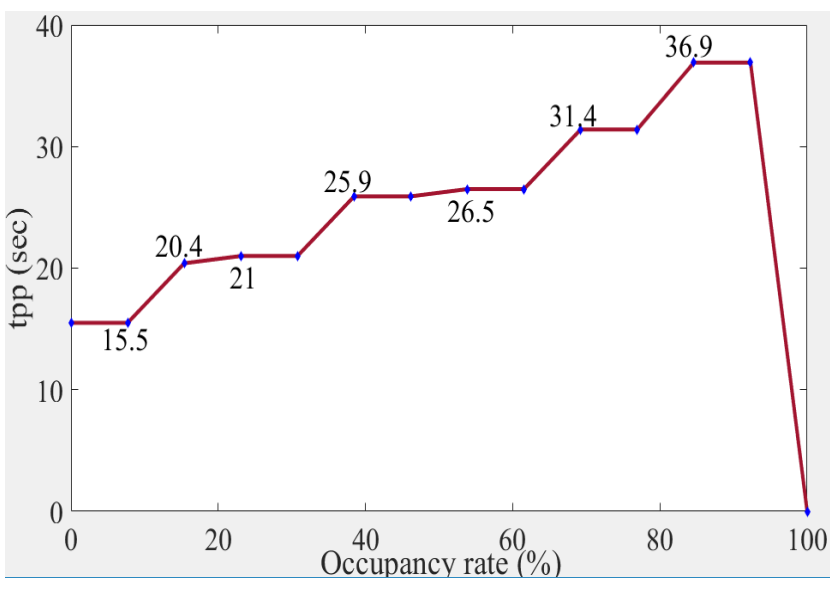

Fig. 16. Changes in the parking time period according to the occupancy rate of parking lots.

The energy that the proposed system consumes to park a vehicle $\left(E_{p p}\right)$ is equal to the sum of the energy which is consumed to take a vehicle from the vehicle acceptance/delivery lot and then park it into the selected parking lot $\left(E_{p r k}\right)$, and the energy which is consumed to return to the starting position of vehicle carrying unit $\left(E_{r s p}\right)$. Since the energy consumed by a system is equal to its operating time multiplied by the power drawn from the source, the energy consumption of the proposed system in the parking process is calculated using the powers and parking time periods measured in the back-and-forth, lift-and-down, horizontal and vertical movements. Accordingly, if the powers measured for the backand-forth $\left(P_{f b}\right)$, lift-and-down $\left(P_{l d}\right)$, horizontal $\left(P_{h}\right)$ and vertical $\left(P_{v}\right)$ movements are written as multipliers in Eq. 5 and Eq. 6, the energy which is consumed to take a vehicle from the vehicle acceptance/delivery lot and park it into the selected parking lot, and the energy which is consumed to return to the starting position of vehicle carrying unit can be obtained as follows, respectively.

$$
\begin{aligned}
E_{p r k}= & P_{v} \cdot\left(N_{f n}-1\right) \cdot t_{v}+P_{h}\left|4-N_{p l n}\right| \cdot t_{h}+3 \cdot P_{f b} \cdot t_{f b}+ \\
& 2 \cdot P_{l d} t_{l d} \\
E_{r s p}= & P_{v} \cdot\left(N_{f n}-1\right) \cdot t_{v}+P_{h \cdot}\left|4-N_{p l n}\right| \cdot t_{h}+P_{f b} \cdot t_{f b}
\end{aligned}
$$

Using both equations, the energy consumed by the system in the parking process is obtained as $E_{p p}=E_{p r k}+E_{r s p}$. Table 7 shows the energy consumptions calculated for the proposed system according to the measured powers in the back-and-forth, lift-and-down, horizontal and vertical movements, and the parking time periods given in table 6 . As in the results of parking time periods, the energy consumptions to reaching the parking lots on the right and left of the vehicle acceptance/delivery lot are also equal to each other. In addition to the results, the parking lots with minimal and maximum energy consumption in the parking process are the same as the ones with the shortest and longest parking time period given in previous results. The total energy consumed in the parking process is obtained as 1829.11 watt.sec in the case that all parking lots of the system are full. This consumption is only valid for the parking process. In the case that the parking exit process is added to this process, the total energy consumed by the system will be twice of this value. 
Table 7. Energy consumptions related to the parking process of a vehicle according to parking lot numbers.

\begin{tabular}{llllllll}
\hline $\begin{array}{l}\text { Par. } \\
\text { lots }\end{array}$ & $\begin{array}{l}\mathbf{P v}_{\text {up }} \\
(\mathbf{W})\end{array}$ & $\begin{array}{l}\mathbf{P v}_{\text {down }} \\
(\mathbf{W})\end{array}$ & $\begin{array}{l}\mathbf{P h} \\
(\mathbf{W})\end{array}$ & $\begin{array}{l}\mathbf{P f b} \\
(\mathbf{W})\end{array}$ & $\begin{array}{l}\mathbf{E}_{\text {apl }} \\
(\mathbf{W} .\end{array}$ & $\begin{array}{l}\mathbf{E}_{\text {rsp }} \\
\mathbf{( W .} \\
\mathbf{s e c})\end{array}$ & $\begin{array}{l}\mathbf{E}_{\mathbf{p p}} \\
\mathbf{( W e c})\end{array}$ \\
\hline 11 & 6.96 & 3.36 & 7.92 & 2.40 & 88.91 & 71.34 & 160.25 \\
12 & 6.96 & 3.36 & 7.92 & 2.40 & 67.13 & 49.56 & 116.69 \\
13 & 6.96 & 3.36 & 7.92 & 2.40 & 45.35 & 27.78 & 73.13 \\
14 & 0.00 & 0.00 & 0.00 & 0.00 & 0.00 & 0.00 & 0.00 \\
15 & 6.96 & 3.36 & 7.92 & 2.40 & 45.35 & 27.78 & 73.13 \\
16 & 6.96 & 3.36 & 7.92 & 2.40 & 67.13 & 49.56 & 116.69 \\
17 & 6.96 & 3.36 & 7.92 & 2.40 & 88.91 & 71.34 & 160.25 \\
21 & 6.96 & 3.36 & 7.92 & 2.40 & 125.10 & 88.81 & 213.92 \\
22 & 6.96 & 3.36 & 7.92 & 2.40 & 103.32 & 67.03 & 170.36 \\
23 & 6.96 & 3.36 & 7.92 & 2.40 & 81.54 & 45.25 & 126.80 \\
24 & 6.96 & 3.36 & 7.92 & 2.40 & 59.76 & 23.47 & 83.24 \\
25 & 6.96 & 3.36 & 7.92 & 2.40 & 81.54 & 45.25 & 126.80 \\
26 & 6.96 & 3.36 & 7.92 & 2.40 & 103.32 & 67.03 & 170.36 \\
27 & 6.96 & 3.36 & 7.92 & 2.40 & 125.10 & 88.81 & 213.92 \\
& & & & $\mathbf{E}_{\text {total }}$ & 1106.08 & 723.02 & 1829.11
\end{tabular}

Fig. 17 depicts the changes in energy that the system consumes when parking a vehicle into the selected parking lot according to the occupancy rate of the parking lots. The graph clearly shows that as the occupancy rate increases, the energy that the system consumes to park a vehicle will also increase. While the energy consumed to park a vehicle is 73.13 watt.sec when the occupancy rate is $0 \%$, this consumption is 213.92 watt.sec when the rate is $85 \%$.

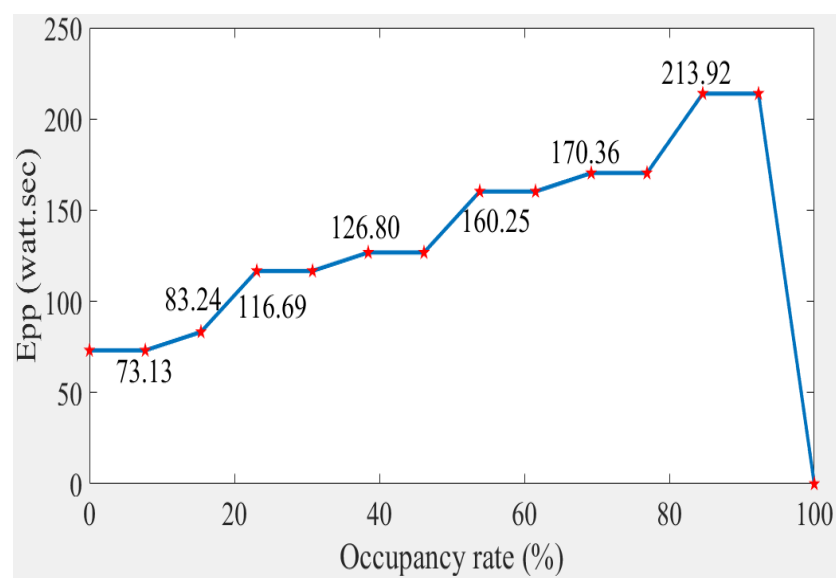

Fig. 17. Changes in energy consumption according to the occupancy rate of the parking lots.

An FL-based decision maker to determine discount rate in the system is used to allow drivers who frequently use the parking lot to pay up to $40 \%$ less parking charges than normal. The FL algorithm determines the discount rate in parking charges by taking into account a yearly the usage frequency (day/year) and usage time (hours/year) of parking lot by a driver. Fig. 18 shows the surface graphic representing the changes in discount rate according to the usage frequency and usage time parameters. It is clearly understood from the graphic that a change in usage frequency and usage time is directly affects the discount rate. The increase in both parameters leads to an increase in the discount rate, while it decreases if the situation is reversed.

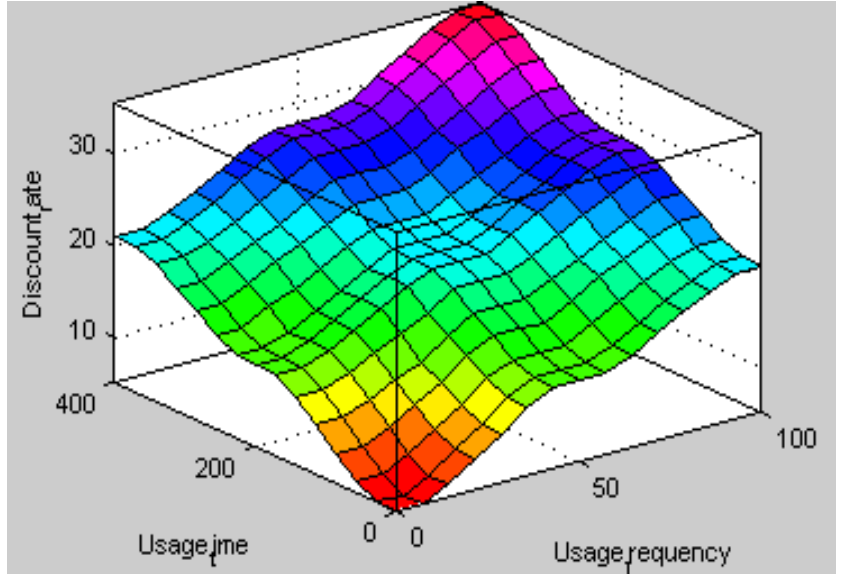

Fig. 18. Changes in discount rate according to the usage frequency and usage time parameters.

A comparison of the classical and automatic parking buildings in accordance with their spaces and volumes are given in Table 8. It can be seen from the data in the table that automatic parking buildings have less space and volume than the classical parking buildings. This will provide significant contributions to the elimination of area-based problems in large urban settlements with narrow land use.

Table 8. Comparison of classical and automatic parking buildings.

\begin{tabular}{lcc}
\hline Property & $\begin{array}{c}\text { Classic } \\
\text { parking } \\
\text { building }\end{array}$ & $\begin{array}{c}\text { Automatic } \\
\text { parking } \\
\text { building }\end{array}$ \\
\hline Number of parking lots (piece) & 600 & 600 \\
Number of floors (piece) & 9 & 13 \\
Land size $\left(\mathrm{m}^{2}\right)$ & 2200 & 820 \\
Surface area $\left(\mathrm{m}^{2}\right)$ & 19600 & 11700 \\
Surface area per Parking lot & 32.67 & 19.50 \\
$\left(\mathrm{~m}^{2} /\right.$ piece) & & \\
Construction volume $\left(\mathrm{m}^{3}\right)$ & 72600 & 30600 \\
\hline
\end{tabular}

\section{Conclusion}

In this study, an automatic parking system that automatically fulfills all operations related to the park process which occur in a multi-stored semi-circular building was designed and its practical application was realized on a prototype. In the proposed system, the OPLS algorithm was used in order to achieve the parking process with the fastest and least energy consumption. The results obtained from the realized prototype showed that the proposed system could perform the parking process quickly and with low energy consumption. It was observed that the performance on the speed and energy consumption increased even more, especially when the occupancy rate of parking lots was less than $50 \%$. The system also provides a low cost parking charges opportunity for drivers who is frequently used the parking lots by putting the FLbased decision-maker determining discount rate into effect. In addition, because the proposed system uses semi-circular building as parking lots, it needs less land space as a place to occupy. As a result, the proposed automatic parking system, thanks to these features, can provide significant contributions to minimize the adverse effects caused by insufficient parking lots especially in urban settlements. 


\section{References}

[1] B. Yıldırım, N. Kuloğlu ve Ö. Güneş, "Otopark sorunu; Elazı̆ğ örneği," 3. Ulaştırma Kongresi, İstanbul, Türkiye, 1995, s. 279-296.

[2] E. K. Güngör, "Konya şehir merkezindeki otopark sorunu ve öneriler," Y.L. Tezi, Selçuk Üniversitesi Fen Bilimleri Enstitüsü, Konya, Türkiye, 2006.

[3] J. Wolff, T. Heuer, H. Gao, M. Weinmann, S. Voit, and U. Hartmann, "Parking monitor system based on magnetic field senso," IEEE in Intelligent Transportation Systems Conference, 2006, pp. 1275-1279.

[4] G. Annosha, S. Lavanya, S. Sivambiga and R. Soundarya, "Embedded based smart car parking system using shared memory," International Journal for Scientific Research and Development, vol. 3, no. 1, pp. 1122-1125, 2015.

[5] V. S. Sahiti, "Automated smart car parking system," International Journal of Computer Engineering and Applications, vol. 11, no. 9, pp. $1-4,2017$.

[6] G. Yan, W. Yang, D. B. Rawat and S. Olariu, "Smart parking: A secure and intelligent parking system," IEEE Intelligent Transportation Systems Magazine, vol. 3, no. 1, pp. 18-30, 2011.

[7] V. P. Thakare and N. A. Chavan, "Performance evaluation of parking guidance and management system using wireless sensor network," International Journal of Recent Technology and Engineering, vol. 1, no. 2, pp. 96-102, 2012.

[8] S. Nagakalyan and B. Raghukumar, "Fabrication of car parking prototype using piezoelectric sensors," International Journal of Mechanical and Mechatronics Engineering, vol. 14, no. 4, pp. 26-30, 2014.

[9] M. S. Yardım ve M. Ağrikli, "Otomatik otoparklar ve türkiye'deki otopark probleminin çözümü için uygulama potansiyeli," 6. Ulaştırma Kongresi TMMOB İş̧aat Mühendisleri Odası İstanbul Şubesi, İstanbul, Türkiye, 2005, s. 363-371.

[10] V. S. Sudha, V. D. Raj, K. R. Sekhar and P. Harika, "GSM based auto secured car parking system," International Journal of Scientific Development and Research, vol. 1, no. 9, pp. 474-477, 2016.

[11]R. Yusnita, F. Norbaya, and N. Basharuddin, "Intelligent parking space detection system based on image processing," International Journal of Innovation, Management and Technology, vol. 3, pp. 232235, 2012.

[12]H. R. Al-Absi, J. D. D. Devaraj, P. Sebastian and Y. V. Voon, "Vision based automated parking system," $10^{\text {th }}$ IEEE International Conference on Information Science, Signal Processing and their Applications, Kuala Lumpur, Malaysia, 2010, pp. 757-760.

[13]K. A. Sunitha, K. Prema, G. S. Deepthi, E. J. E. Belinda and N. S. Kumar, "Fuzzy based automatic multi-level vehicle parking using lab view," IEEE International Conference on Frontiers in Automobile and Mechanical Engineering, Chennai, India, 2010, pp. 363-367.

[14] S. M. Bhadkumbhe, A. Narayan, D. Narayan, S. Shaikh and Y. Kunjir, "Efficient automated smart parking system using iot technologies," Imperial Journal of Interdisciplinary Research, vol. 3, no. 4, pp. 16211624, 2017.

[15]F. Islam, M. Adil and S. A. Alvi, "PLC based automatic intelligent car parking system," International Journal of Computer Theory and Engineering, vol. 9, no. 1, pp. 53-57, 2017. 